\title{
Ex vivo differentiation of human bone marrow- derived stem cells into neuronal cell-like lineages
}

This article was published in the following Dove Press journal:

Journal of Neurorestoratology

27 June 2016

Number of times this article has been viewed

\author{
Adeeb Al-Zoubi ${ }^{1,2}$ \\ Feras Altwal ${ }^{3}$ \\ Farah Khalifeh ${ }^{2}$ \\ Jamil Hermas ${ }^{4}$ \\ Ziad Al-Zoubi ${ }^{5}$ \\ Emad Jafar ${ }^{5}$ \\ Mohammed El-Khateeb ${ }^{6,7}$ \\ 'Department of Surgery, University of \\ Illinois College of Medicine at Peoria, Peoria, \\ IL, USA; ${ }^{2}$ Stem Cells of Arabia, Amman, \\ Jordan; ${ }^{3}$ Department of Neuroscience, \\ School of Graduate and Postdoctoral \\ Studies, Rosalind Franklin University of \\ Medicine and Science, North Chicago, \\ IL, USA; ${ }^{4}$ Stem Cell Division, Al-Yamama \\ Company, ${ }^{5}$ Jordan Orthopedic and Spinal \\ Center, ${ }^{6} \mathrm{National}$ Center for Diabetes, \\ Endocrinology and Genetics, ${ }^{7}$ Department of \\ Pathology, Faculty of Medicine, University of \\ Jordan, Amman, Jordan
}

\section{Video abstract}

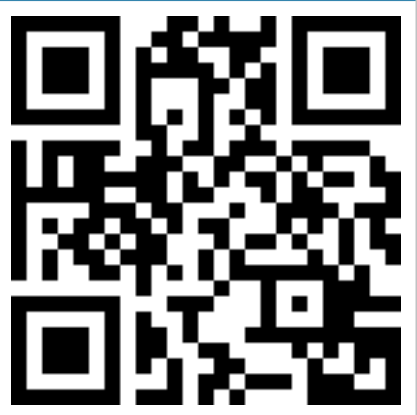

Point your SmartPhone at the code above. If you have a QR code reader the video abstract will appear. Or use: http://youtu.be/vgRIJPSQKZ8

Correspondence: Adeeb Al-Zoubi Stem Cells of Arabia, 3rd Floor,

246 Al-Medina Al-Munawara Street, PO Box 2828,Amman II953, Jordan

Tel +96265692 I I I

Fax +962 65692114

Email adeebalzoubi@stemcellsarabia.net
Background: Methods to obtain safe and practical populations of stem cells (SCs) at a clinical grade that are able to differentiate into neuronal cell lineages are yet to be developed. In a previous study, we showed that mouse bone marrow-derived SCs (BM-SCs) differentiated into neuronal cell-like lineages when put in a neuronal-like environment, which is a special media supplemented with the necessary growth factors needed for the differentiation of SCs into neuronal cell-like lineages.

Aim: In this study, we aim to assess the potentials of adult human CD34+ and CD133+ SCs to differentiate into neuronal cell-like lineages ex vivo when placed in a neuronal-like microenvironment.

Methods: The neuronal-like microenvironment was created by culturing cells in nonhematopoietic expansion media (NHEM) supplemented with growth factors that favor differentiation into neuronal cell lineages (low-affinity nerve growth factor [LNGF], mouse spinal cord extract [mSpE], or both). Cultured cells were assessed for neuronal differentiation by cell morphologies and by expression of GFAP.

Results: Our results show that culturing unpurified human BM-derived mononuclear cells (hBM-MNCs) in NHEM+LNGF+mSpE did not lead to neuronal differentiation. In contrast, culturing of purified CD34+ hBM-SCs in NHEM+LNGF+mSpE favored their differentiation into astrocyte-like cells, whereas culturing of purified CD133+ hBM-SCs in the same media favored their differentiation into neuronal-like cells. Interestingly, coculturing of CD34+ and CD133+hBM-SCs in the same media enhanced the differentiation into astrocyte-like cells and neuronal-like cells, in addition to oligodendrocyte-like cells.

Conclusion: These results suggest that a mixture of purified CD34+ and CD133+ cells may enhance the differentiation into neuronal cell-like lineages and give broader neuronal cell lineages than when each of these cell types is cultured alone. This method opens the window for the utilization of specific populations of hBM-SCs to be delivered in a purified form for the potential treatment of neurodegenerative diseases in the future.

Keywords: cell therapy, neurodegenerative disease, neurons, astrocyes, CD34+, CD133+, MACS

\section{Introduction}

In recent years, neurons and glial cells have been successfully generated from various types of stem cells (SCs) such as adult neural SCs, mesenchymal SCs (MSCs), embryonic SCs (ESCs), and induced pluripotent SCs. ${ }^{1,2}$ There is still ongoing extensive research to develop SC transplantation therapies for neurological diseases. Strong evidence has come forth of the potential of various SC populations to induce 
regeneration in animal models of Alzheimer's disease, ${ }^{3}$ multiple sclerosis, ${ }^{4}$ Parkinson's disease,${ }^{5}$ Huntington's disease, ${ }^{6}$ and spinal cord injury (SCI) over the last decade. Some of these reports were already upgraded to clinical trials. On another level, ESCs have been grafted in patients with SCIs for the first human trials of human ESC research according to a preliminary authorization by the US Food and Drug Administration (FDA) to prove whether the use of such cell types is safe or not. ${ }^{7}$ But many concerns surround the ESC implication in clinical trials, majorly due to the deep-rooted observation that ESCs can, in fact, induce the formation of teratomas in studied animal models. ${ }^{8}$ These testimonials suggest that bone marrow-derived SCs (BM-SCs) and especially autologous SCs are more appropriate for these therapies. This is mainly because SCs exist in higher numbers in the BM, which is an easily accessible source for SCs; autologous SCs are nonimmunogenic and have high self-renewal rates; and the BM in most patients with neurological diseases is intact and unaffected by the disease. ${ }^{9}$

Recent studies have elucidated that cells with similar functional and morphological features to mesenchymal BM-SCs can be isolated from various other organs and tissues such as the peripheral blood and adipose tissue. ${ }^{10}$ Even though MSCs arise from different origins, these cells possess cellular physiological properties that are typically associated with SCs, such as self-renewability and high potentiality to differentiate into specialized cell lineages including neuronal cell lineages. ${ }^{11}$

BM-SC therapy represents a valid alternative to repair and replace damaged or lost neurons. Attempts to differentiate SCs into neuronal cell lineages ex vivo using different combinations of growth/differentiation factors have been made. ${ }^{12}$ However, using these methods does not represent the true physiological conditions of the injured central nervous system (CNS) tissue in humans.

Previously, we reported a method for the ex vivo differentiation of mouse SCs into tissue-specific cell types, including neuronal lineages. ${ }^{13}$ In this study, we differentiated adult human BM-derived SCs (hBM-SCs) ex vivo into cells of neuronal lineages. We formulated and used mouse spinal cord extract $(\mathrm{mSpE})$, which showed stimulatory effects on the differentiation of hBM-SCs into neuronal cell lineages. Additionally, our results showed magnified neuronal differentiation capabilities of purified CD34+ and CD133+hBM-SCs when cultured in media supplemented with $\mathrm{mSpE}$, compared to unpurified hBM mononuclear cells (hBM-MNCs). CD34+ SCs showed a preferential differentiation into astrocyte-like cells, whereas CD133+ SCs preferentially differentiated into neuronal-like cells. Interestingly, CD34+/CD133+ mixed cultures displayed even higher neuronal differentiation capabilities, in addition to generating oligodendrocyte-like cells which were seen only in the mixed cultures. The differences in differentiation potentials between CD34+ and CD133+ SCs presented in this study are of high significance in the identification of suitable hBM-SC types that may have the capability to differentiate into astrocytes and neurons, respectively, with possible applications in the treatment of diverse neuronal diseases. This model was similarly applied in a study published in 2014, in which we reported treating patients with chronic complete SCIs by utilizing autologous, purified CD34+ and CD133+ SCs. The study focused on the safety and efficacy of transplanting unmanipulated, autologous, purified SCs in treated patients during a 5-year follow-up period. ${ }^{14}$

\section{Materials and methods Preparation of bone marrow mononuclear cells (BM-MNCs)}

The Ethical Committee of Stem Cells of Arabia approved the use of human bone marrow in this study. After obtaining written informed consents from all of the donors, samples $(20 \mathrm{~mL})$ of fresh BM were used. MNCs were collected by density-gradient centrifugation using Ficoll-Paque (SG 1.077; GE Pharmacia, North Highland, CA, USA) according to previously published protocols. ${ }^{14}$ Briefly, $15 \mathrm{~mL}$ of Ficoll-Paque solution was aliquoted in $50 \mathrm{~mL}$ conical tubes, and topped with $35 \mathrm{~mL}$ of aspirated BM (diluted 1:2 with phosphate-buffered saline $[\mathrm{PBS}]+$ ethylenediaminetetraacetic acid [EDTA]). Samples were centrifuged at $450 \times g$ for 40 minutes at room temperature (RT). The upper layer containing the plasma was discarded, and the buffy coat containing the MNCs was collected, diluted with PBS, and centrifuged at $350 \times g$ for 15 minutes at RT to ensure the removal of the remaining plasma and Ficoll-Paque remnants. After removing the supernatant, the MNCs were diluted with PBS and centrifuged at $200 \times g$ for 20 minutes at RT. The pellet was then resuspended in $10 \mathrm{~mL}$ of PBS, and samples were taken for cell count and flow cytometric analyses.

\section{Selection of CD34+/CDI33+ SCs by magnetic labeling and separation (MACS)}

CD34+ and CD133+ SCs were purified from MNCs by magnetic separation using magnetic-activated cell sorting (MACS) technology (MACS ${ }^{\circledR}$ technology; Miltenyi Biotec, $\mathrm{GmbH}$, Bergisch Gladbach, Germany) according to the manufacturer's protocol. Briefly, BM-MNCs were centrifuged, 
supernatant discarded, and the pellet diluted in $80 \mu \mathrm{L}$ of PBS $/ 10^{7}$ cells. A volume of $20 \mu \mathrm{L}$ of either CD34 or CD133 MicroBeads (Miltenyi Biotec) (or both combined) was added for up to $10^{7}$ of MNCs. Cells were then mixed and incubated with the MicroBeads for 15 minutes at $4^{\circ} \mathrm{C}$. After incubation, cells were washed twice with $10 \mathrm{~mL}$ of PBS and centrifuged at $300 \times g$ for 15 minutes at $4^{\circ} \mathrm{C}$. Cells were then diluted with $500 \mu \mathrm{L}$ of PBS for up to $10^{7}$ cells. Target cell populations were purified using the autoMACS ${ }^{\circledR}$ Pro system (Miltenyi Biotec) and running the POSSELDS program. After separation, two fractions were obtained, the positive fraction containing CD34+, CD133+, or both CD34+/CD133+ depending on the MicroBeads used, and negative fraction containing CD34-/ CD133- cells. Cells were then counted, and the purities and viabilities were analyzed using flow cytometry.

\section{Flow cytometric analyses}

MNCs in the original BM fraction and in the purified populations (positive and negative fractions) were assessed to determine the percentages of CD34+ and CD133+ SC populations using the MACSQuant flow cytometer. Fractions obtained from the aforementioned experiment were washed as described in the previous section, and resuspended in $500 \mu \mathrm{L}$ of PBS buffer for flow cytometry analyses. To allow for multiple analyses, cells in all fractions were costained with anti-CD13-phycoerythrin and anti-CD34-fluorescein isothiocyanate. Flow cytometry analyses were performed according to preset parameters specifically designed for this study.

\section{Culture of BM-SCs and MNCs for ex vivo differentiation}

After magnetic purification of SCs, unpurified BM-MNCs and each purified SC fraction were cultured in nonhematopoietic expansion media (NHEM) (Miltenyi Biotec) supplemented with $10 \%$ fetal calf serum (Bio Products Laboratory, Hertfordshire, UK), $100 \mathrm{U} / \mathrm{mL}$ penicillin, $100 \mathrm{mg} / \mathrm{mL}$ streptomycin, and $100 \mathrm{mg} / \mathrm{mL}$ Fungizone (Gibco 15140, Thermo Fisher Scientific, Waltham, MA, USA). To allow for the differentiation of SCs into neuronal-like cells, we used NHEM supplemented with either low-affinity nerve growth factor (LNGF) (Miltenyi Biotec) or our previously described $\mathrm{mSpE}$. Briefly, the extract was prepared by grinding mouse spinal cord, filtering the extract, and resuspending it in $5 \mathrm{~mL}$ NHEM, and incubating it overnight at $37^{\circ} \mathrm{C}$ in a $\mathrm{CO}_{2}$ incubator. After 12 hours, the extract was centrifuged at $350 \times \mathrm{g}$ for 15 minutes at RT, and the supernatant was filtered with a $0.2 \mu \mathrm{M}$ filter, aliquoted, and stored at $-20^{\circ} \mathrm{C}$ to be used in SC culture every 3-5 days.

\section{Immunocytochemistry}

Cultured BM-SCs were fixed with 4\% paraformaldehyde, incubated with primary monoclonal antibodies for GFAP, neurofilament protein (NFP), and S100 (Dako Denmark A/S, Glostrup, Denmark) overnight at $4^{\circ} \mathrm{C}$, incubated with secondary antibodies for 1 hour, followed by exposure to avidin-biotin complex for 1 hour $\left(25^{\circ} \mathrm{C}\right)$. Diaminobenzidine served as a chromogen. Cells were imaged under a fluorescent microscope (Nikon Corporation, Tokyo, Japan).

\section{Results \\ Flow cytometry analysis}

We collected an average of $213 \times 10^{6}\left( \pm 55 \times 10^{6}\right)$ MNCs from $20 \mathrm{~mL}$ of BM. The analysis of the original cell fraction (MNCs from the BM) showed $1.1 \%$ of CD34+ cells and $0.8 \%$ of CD133+ cells (Figure 1A, C, and E). After purifying the cells using the MACS technique, the analyses showed $98.9 \%$ of CD34+ cells and 83.2\% of CD133+ cells (Figure 1B, D, and F). These results indicated high efficiency of using the magnetic cell separation system in purifying specific populations of SCs.

\section{MACS and microscopic imaging}

The average yield of CD34+ SCs was $2.3 \times 10^{6}\left( \pm 0.6 \times 10^{6}\right)$ cells, whereas the yield of CD133+ SCs was $1.7 \times 10^{6}$ $\left( \pm 0.7 \times 10^{6}\right)$ cells. The purified fractions of cells were imaged under the inverted microscope for the assessment of the initial morphology for comparison purposes and a better understanding of the development of cell morphology during culture. The MNC fraction does not show an obvious morphology upon imaging (Figure 2A), in comparison to the purified $\mathrm{CD} 34+$ fraction which reveals a clear image of round cells, with a considerable count (Figure 2B). Additionally, the CD133+ fraction also shows a clear image of round cells, but with less cell count (Figure 2C). The double positive fractions (CD34+/CD133+) reveal a clear image of vital round cells (Figure 2D), which resembles the cells shown in Figure $2 \mathrm{~B}$ and $\mathrm{C}$.

\section{Culture of BM-SCs and MNCs for ex vivo differentiation}

Culture-differentiated BM-derived cells are shown in Figure 3, which clearly demonstrates undifferentiated BM-MNCs. Purified CD34+, CD133+, and CD34+/CD133+SCs were separately cultured in NHEM alone (Figure 3A-D); NHEM+ $\mathrm{mSpE}$ (Figure 3E-H); NHEM+LNGF (Figure 3I-L); or NHEM+mSpE+LNGF (Figure 3M-P). Typical representatives of cells with different neuronal cell lineage-like 

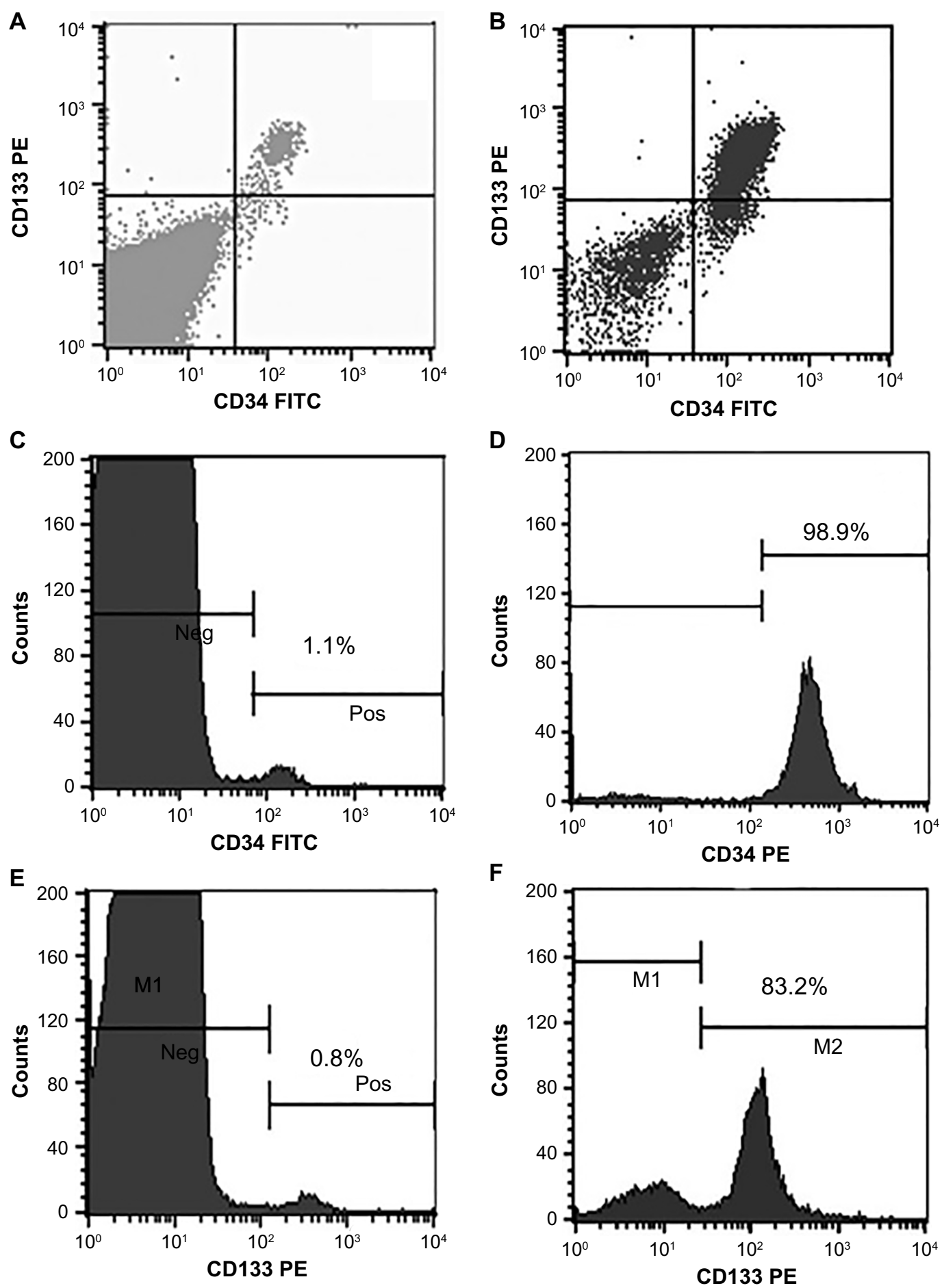

Figure I Flow cytometry analyses.

Notes: Shown are the percentages of CD34+ and CDI33+ stem cells in bone marrow mononuclear cells (A, C, and E) and in purified CD34+ stem cells (D), purified CDI33+ stem cells (F), and both purified CD34+ and CDI33+ stem cells (B). In histograms C, D, E, and F, MI is a marker for cells that do not express CD34 or CDI33 (CD34- and CDI33-), whereas M2 is a marker for cells that do express these markers (CD34+ and CDI33+ cells).

Abbreviations: FITC, fluorescein isothiocyanate; Neg, nevative; Pos, positive; PE, phycoerythrin.

morphologies that were obtained in each culture condition are shown in Figure 3. Representatives of MNCs are shown in Figure 3A-E, I, and M; astrocytes are shown in Figure $3 \mathrm{~F}, \mathrm{~J}$, and N; neurons are shown in Figure 3G, K, and $\mathrm{O}$; and oligodendrocytes are shown in Figure $3 \mathrm{H}, \mathrm{L}$, and $\mathrm{P}$.
Based only on morphology, higher percentages of human CD34+ BM-SCs differentiated into astrocyte-like cells when placed in the neuronal differentiation media. Additionally, when placed in the same media, human CD133+ BM-SCs showed more preferential differentiation into neuronal-like 

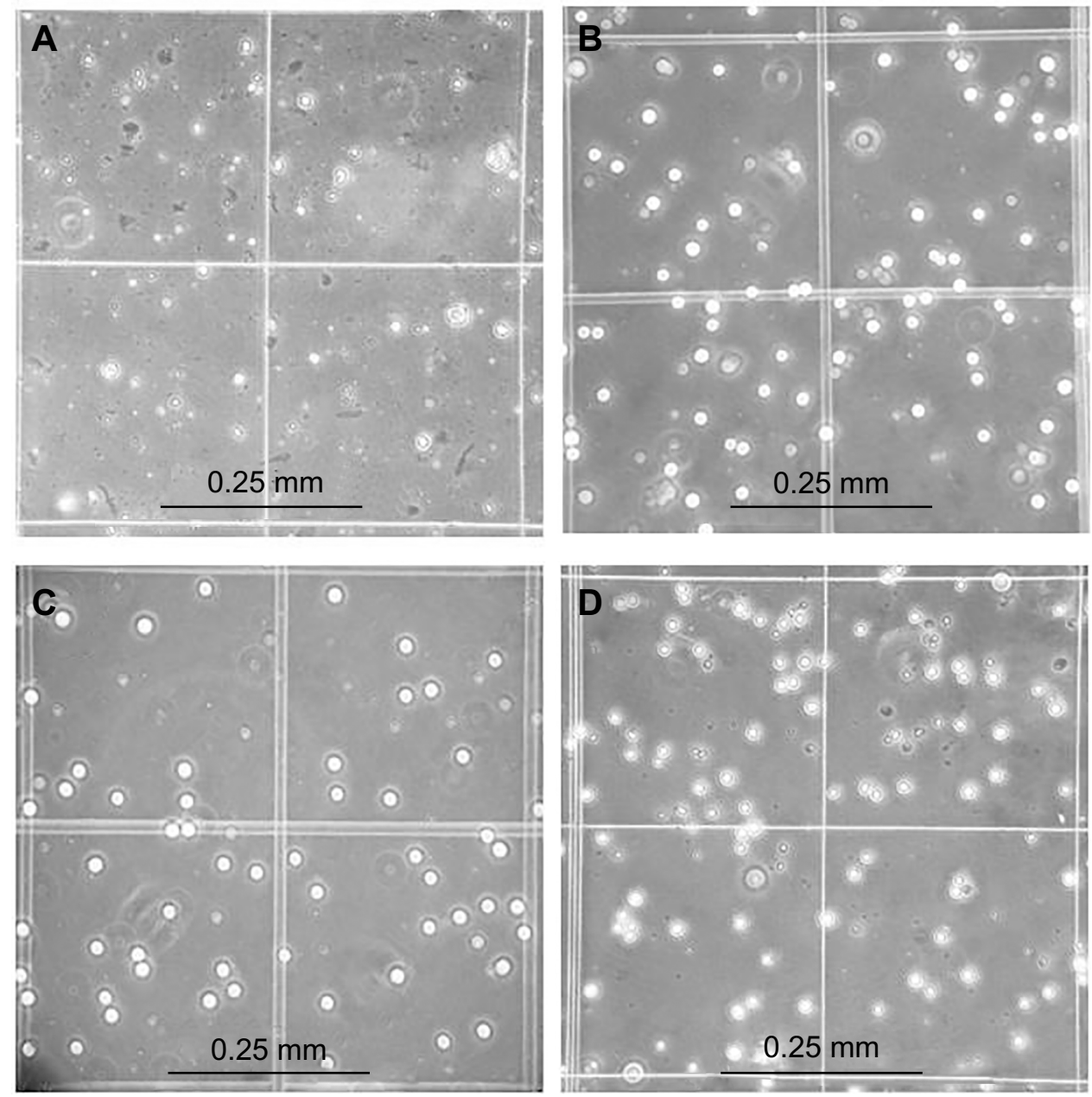

Figure 2 Microscopic images of cells before and after magnetic purification.

Notes: (A) Bone marrow mononuclear cells; (B) Purified CD34+ stem cells; (C) Purified CDI33+ stem cells; and (D) Purified CD34+ and CDI33+ stem cells. Cells were viewed under $40 \times$ magnification.

cells, whereas CD34+/CD133+ BM-SCs produced more oligodendrocyte-like cells than other culture conditions.

\section{Immunohistochemistry}

Immunohistochemistry of cells was carried out with antibodies against GFAP, S100, and NFP. GFAP, S100, and NFP-stained MNCs are shown in Figure 4A, E, and I; purified CD34+ cells are shown in Figure 4B, F, and J; purified CD133+ cells are shown in Figure 4C, G, and K; and purified CD34+/CD133+ cells are shown in Figure 4D, H, and L. Accordingly, astrocytes stained positive for GFAP and S100 (Figure 4B and F) and negative for NFP. Neurons stained positive for NFP and S100 (Figure 4K and G) and negative for GFAP (Figure 4C). Oligodendrocytes stained positive for S100 (Figure 4H) and negative for GFAP and NFP. Figure 5 shows the comparison between positive staining in primary and secondary neurons (Figure 5A and $\mathrm{B}$ ) and negative staining in oligodendrocytes (Figure 5C). The efficiencies of MNCs and CD34+, CD133+, and CD34+/CD133+ cells to differentiate into neuronal cell-like types were measured by counting the numbers of cells that stained positive for the earlier antibodies, over all cells counted in that field. Assessments of these efficiencies are presented in Table 1. The highest efficiencies for differentiation into all three neuronal cell-like types (astrocytes, neurons, and oligodendrocytes) were seen when CD34+/CD133+ cells were cultured in NHEM+LNGF+mSpE, compared with other culture conditions (NHEM, NHEM+LNGF, and NHEM+mSpE).

\section{Timeline of ex vivo differentiation of purified CDI33+ SCs into neurons}

Herein, we present the complete timeline of the differentiation of purified BM-derived CD133+ SCs cultured in NHEM supplemented with $\mathrm{mSpE}$ for defined periods of time to allow for differentiation into neuronal cell lineages. CD133+ SCs were cultured for 4 days (Figure 6A), 8 days (Figure 6B), 

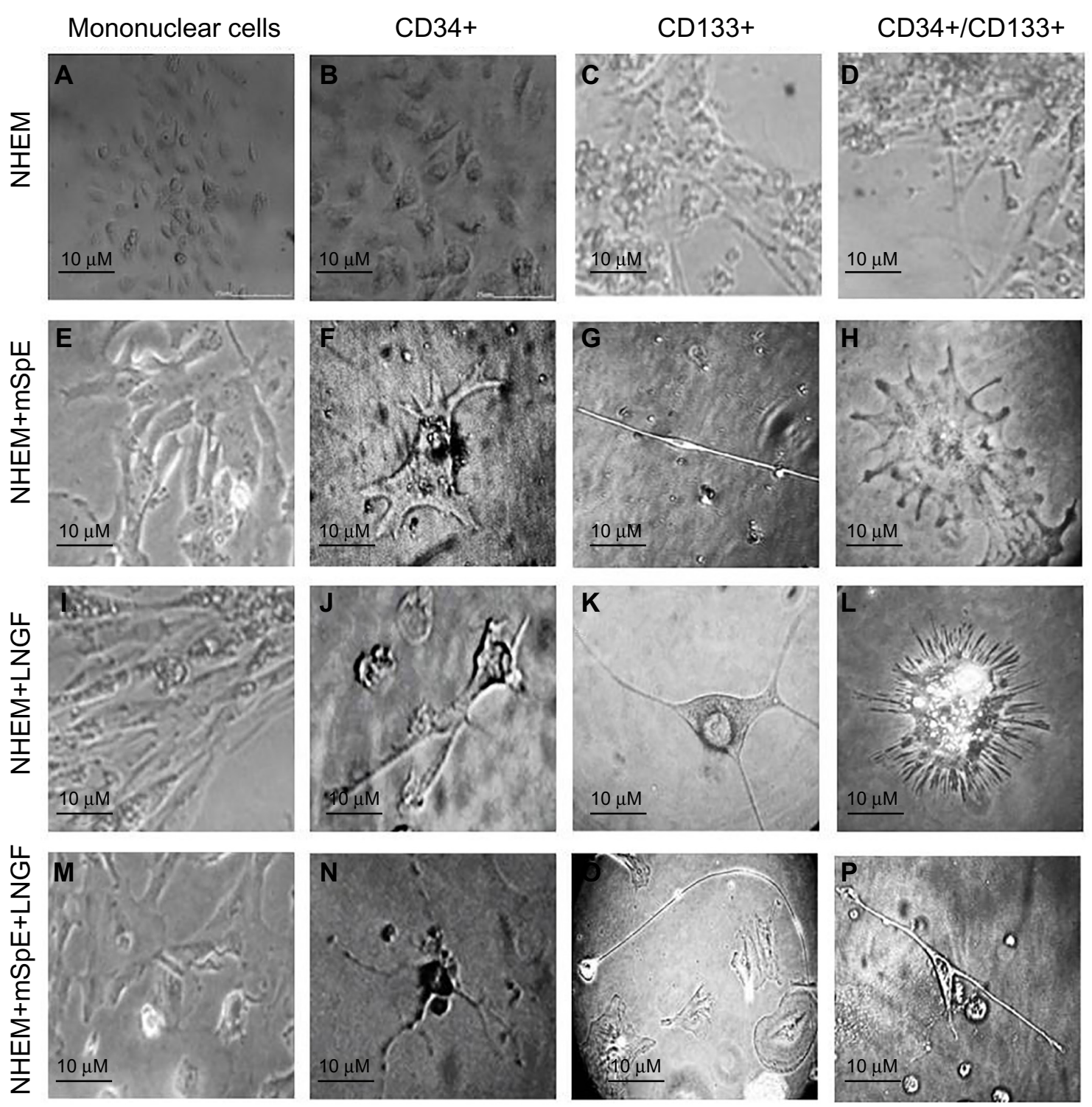

Figure 3 Culture-differentiated bone marrow-derived cells.

Notes: Undifferentiated bone marrow mononuclear cells, and purified CD34+, CDI33+, and CD34+/CDI33+ stem cells were separately cultured in NHEM alone (A-D), $\mathrm{NHEM}+\mathrm{mSpE}(\mathbf{E}-\mathbf{H}), \mathrm{NHEM}+\mathrm{LNGF}(\mathbf{I}-\mathbf{L})$, or NHEM+mSpE+LNGF (M-P). Shown are cells with different neuronal cell lineage morphologies: typical mononuclear cells

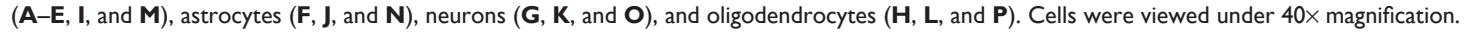

Abbreviations: NHEM, nonhematopoietic expansion media; mSpE, mouse spinal cord extract; LNGF, low-affinity nerve growth factor.

12 days (Figure 6C), 16 days (Figure 6D), and 20 days (Figure $6 \mathrm{E}$ and $\mathrm{F}$ ). The figure clearly shows axonal extension (Figure $6 \mathrm{~A}-\mathrm{D}$ ) and formation of a bipolar neuron (Figure 6F) and a multipolar neuron (Figure 6E).

\section{Discussion}

Recent reports have demonstrated that adult BM-SCs represent feasible candidates in tissue repair with relative ease in harvest and isolation. ${ }^{10} \mathrm{BM}$-SCs have been useful to patients with hematological malignancies and other diseases for the past few decades. MSCs or BM-SCs offer the advantage of minimizing immune reactions to negligible levels because, as shown previously, these can be derived from the patients themselves. Several promising studies have reported the differentiation of MSCs into neural SC lineages. But, the full evidence of efficient functional neurons derived from MSCs is not yet demonstrated. Therefore, the concept of possible differentiation into cells with neurological-like phenotype is presented in this study. Nevertheless, it now appears more feasible and applicable to convert fibroblasts directly into neurons with new and improved technologies such as induced pluripotent SCs. ${ }^{15,16}$ 

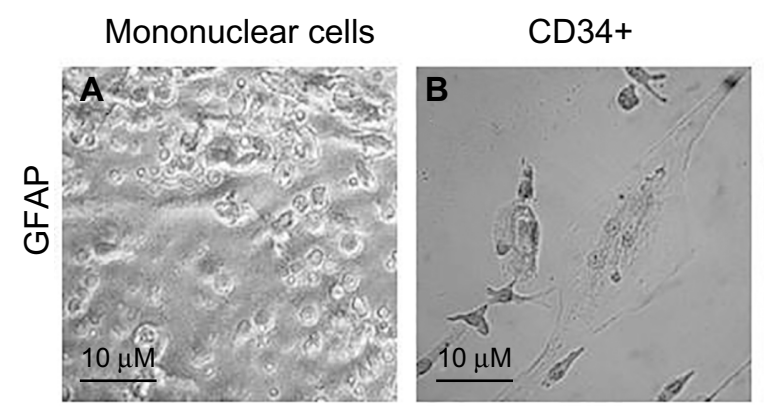

CD133+
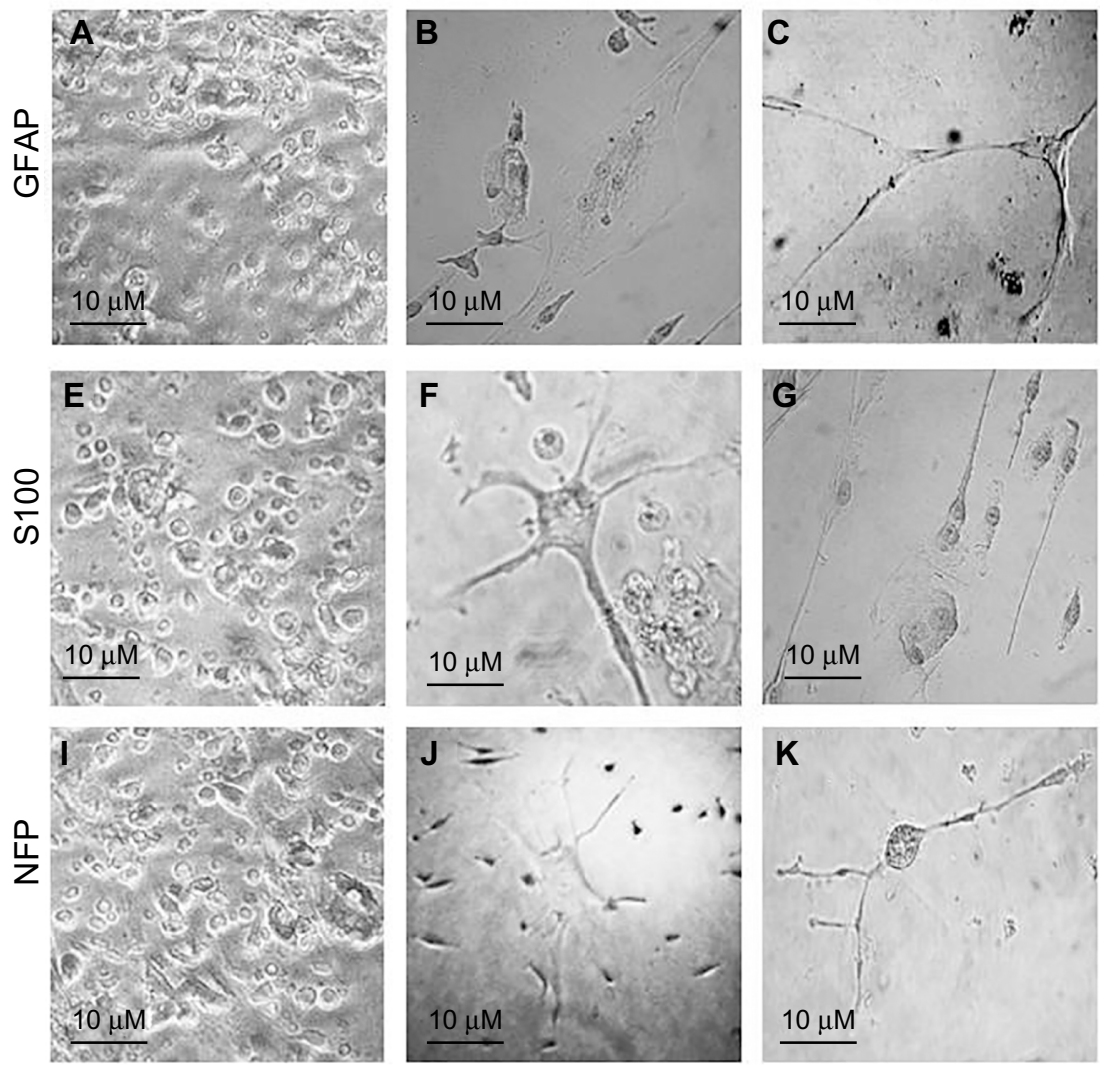
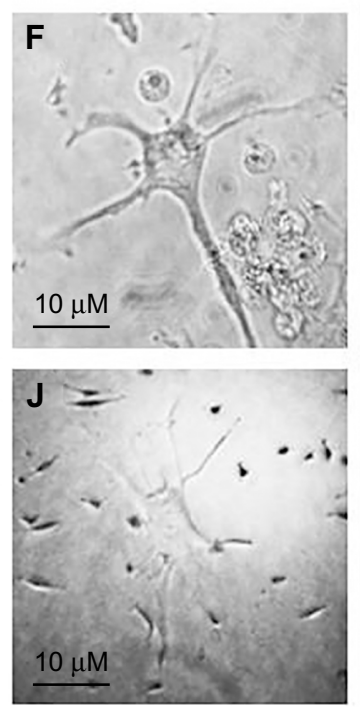
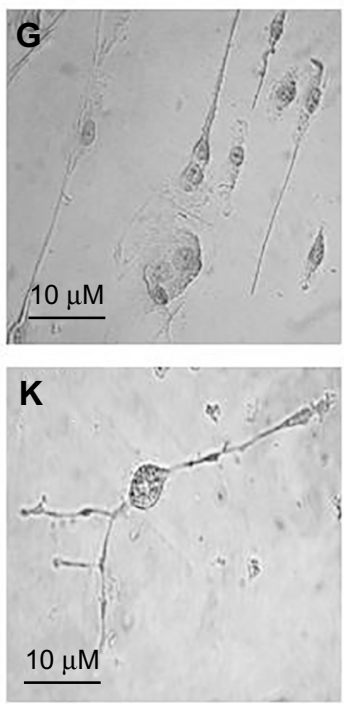

CD34+/CD133+
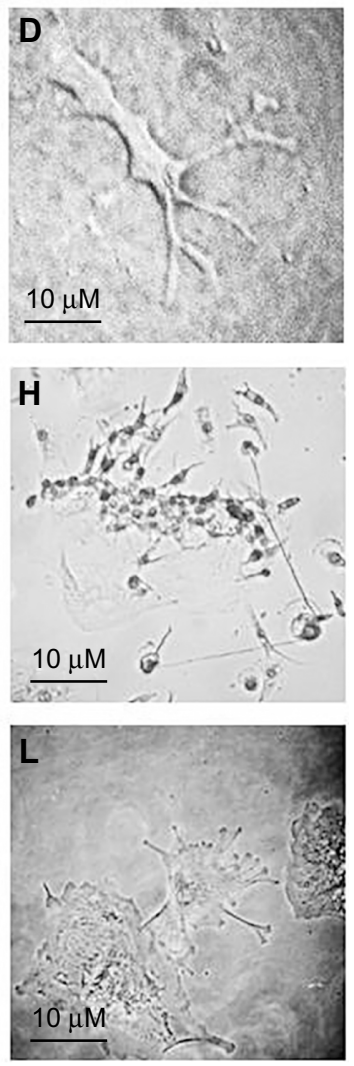

Figure 4 Immunocytochemistry of cells with antibodies against GFAP, SI00, and NFP.

Notes: Shown are mononuclear cells $(\mathbf{A}, \mathbf{E}$, and I), and purified CD34+ (B, F, and J), CDI33+ (C, G, and K), and CD34+/CDI33+ (D, H, and L) stem cells. Cells were culture-differentiated using different conditions, and then stained with antibodies against GFAP (A-D), SI00 (E-H), or NFP (I-L). Astrocytes are clearly seen positive for GFAP (B) and SI00 (F), and negative for NFP (J). Neurons stained positive for NFP (K) and SI00 (G), and negative for GFAP (C). Oligodendrocytes stained positive for SI00 (H) and were negative for GFAP (D) and NFP (L). Cells were viewed under 40× magnification.

In this study, we assessed the efficiencies of MNCs and CD34+, CD133+, and CD34+/CD133+ cells to differentiate into neuronal-like cell types (Table 1). Although the efficiencies for differentiation into neuronal-like cell types were low $(<11 \%$ for any given antibody), there were clear differences between different SC types when cultured in different media conditions (Table 1). However, accurate differentiation efficiencies of MNCs and CD34+, CD133+, and CD34+/ $\mathrm{CD} 133+$ cells to generate neuronal-like cell types cannot be determined via immunohistochemistry staining procedures, due to the limitation of the procedure, unavailability of facilities, and the need for much larger cell numbers for each experimental variable. In order to accurately assess the percentages of cells that can differentiate into any cell type,

Table I Assessment of cellular potentialities to differentiate into neuronal cell types

\begin{tabular}{|c|c|c|c|c|c|c|c|c|c|c|c|c|}
\hline \multirow{2}{*}{$\begin{array}{l}\text { Culture media } \\
\text { condition }\end{array}$} & \multicolumn{3}{|c|}{ BM-MNCs } & \multicolumn{3}{|l|}{ CD34+ } & \multicolumn{3}{|c|}{ CDI33+ } & \multicolumn{3}{|c|}{ CD34+/CDI33+ } \\
\hline & GFAP & $\mathbf{S 1 0 0}$ & NFP & GFAP & S100 & NFP & GFAP & S100 & NFP & GFAP & S100 & NFP \\
\hline NHEM & $<1 \%$ & $<1 \%$ & $<1 \%$ & $<1 \%$ & $<1 \%$ & $<1 \%$ & $<1 \%$ & $<1 \%$ & $<1 \%$ & $<1 \%$ & $<1 \%$ & $<1 \%$ \\
\hline NHEM+LNGF & $<1 \%$ & $<1 \%$ & $<1 \%$ & $6 \%$ & $<5 \%$ & $<1 \%$ & $<1 \%$ & $5 \%$ & $4 \%$ & $<3 \%$ & $<3 \%$ & $<2 \%$ \\
\hline $\mathrm{NHEM}+\mathrm{mSp} \mathrm{E}$ & $<1 \%$ & $<1 \%$ & $<1 \%$ & $7 \%$ & $<4 \%$ & $<1 \%$ & $>1 \%$ & $5 \%$ & $5 \%$ & $<3 \%$ & $5 \%$ & $<3 \%$ \\
\hline $\mathrm{NHEM}+\mathrm{LNGF}+\mathrm{mSpE}$ & $<1 \%$ & $<1 \%$ & $<1 \%$ & $5 \%$ & $10 \%$ & $<1 \%$ & $<3 \%$ & $8 \%$ & $6 \%$ & $5 \%$ & $11 \%$ & $7 \%$ \\
\hline
\end{tabular}

Notes: BM-MNCs, CD34+, CDI33+, and CD34+/CDI33+ cells were cultured and were assessed for expression of GFAP, SI00, and NFP. The percentages shown in the table represent the number of cells that stained positive with respective antibody for the given target marker protein, over all the cells counted under that field under the microscope. At least 50 cells were counted in each variable that were spread in different fields.

Abbreviations: NHEM, nonhematopoietic expansion media; LNGF, low-affinity nerve growth factor; mSpE, mouse spinal cord extract; BM-MNCs, bone marrow mononuclear cells. 


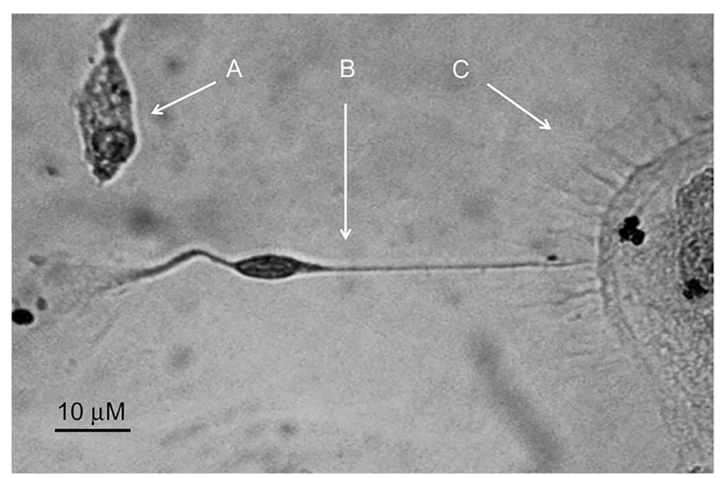

Figure 5 Combination of cells with neurofilament protein (NFP) staining. Notes: Shown are (A) primary and (B) secondary neurons with positive NFP staining, and (C) oligodendrocytes with negative NFP staining. Cells were viewed under $40 \times$ magnification. a more rigorous experiment and different detection and calculation methods, such as flow cytometry, should be used.

Besides the demand of functional differentiation, one may ask: is it possible for these differentiated cells to deliver trophic maintenance to damaged neurons? And, could the transplantation of such cells into the CNS, as done in some centers, affect the final outcome by generating new functional neurons at the site of transplantation?

Our results agree with previously published studies, which showed that human adult CD34+ cells can differentiate into a neural phenotype in the presence of astrocytes. ${ }^{14}$ On the other hand, a retrospective study on brain autopsy samples of BM-transplanted patients showed the presence
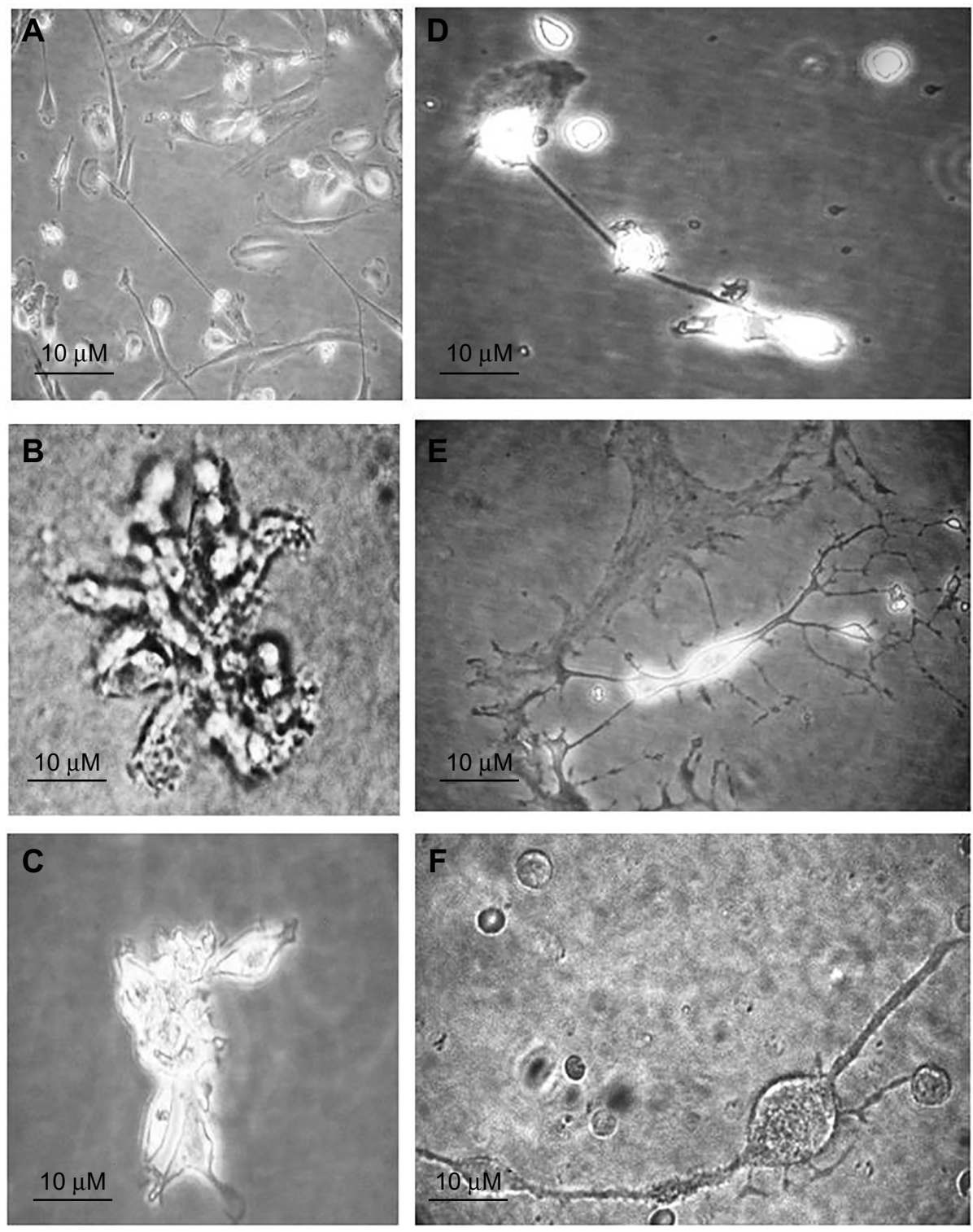

Figure 6 Timeline of ex vivo differentiation of purified CDI33+ stem cells into neurons.

Notes: Purified bone marrow-derived CDI33+ stem cells were cultured in NHEM supplemented with mSpE for defined periods of time to allow for differentiation into neuronal cell lineages. CDI33+ stem cells were cultured for 4 days (A), 8 days (B), 12 days (C), 16 days (D), and 20 days (E and $\mathbf{F})$. This figure clearly shows axonal extension $(\mathbf{A}-\mathbf{D})$ and formation of a bipolar neuron $(\mathbf{F})$ and a multipolar neuron (E). Cells were viewed under $40 \times$ magnification.

Abbreviations: NHEM, nonhematopoietic expansion media; $\mathrm{mSpE}$, mouse spinal cord extract. 
of new hematopoietic SC (HSC)-derived neurons only in patients surviving for a long time period..$^{15}$ Considered as a whole, these results propose that differentiation of HSCs into neurons is an uncommon event in the adult brain, but may be stimulated more commonly by a particular microenvironment present in injured tissues.

Our results support this suggestion, since CNS disease or injury is usually associated with astrocytosis. The activation of astrocytes should regulate the appropriate setting required to stimulate HSC differentiation into neural cells. In this report, we utilized an ex vivo model in an attempt to recreate that microenvironment. Additionally, our previous studies showed that, in this ex vivo experiment, astrocytes proliferate and produce cytokines and other factors. More recent data demonstrated the effects of intravenous human umbilical cord blood CD34+ SCs in experimentally induced parkinsonian mice. CD34+ cells ameliorated motor, biochemical, and histological deficits in parkinsonian mice, and these effects were superior to those produced by levodopa which is promising for the treatment of Parkinson's disease. ${ }^{17}$ Another study showed that CD133+ SCs from amyotrophic lateral sclerosis (ALS) patients incubated for 48 hours in a neuroinduction medium increased the detection of neuronal proteins such as Nestin, $\beta$-tubulin III, neuronal-specific enolase, and GFAP, concluding that CD133+ SCs from ALS patients, like the SCs of healthy individuals, are capable of differentiating into preneuron cells. ${ }^{18}$

Moreover, the proposed model was proven in a previously published study, ${ }^{14}$ in which we presented for the first time the treatment of complete SCIs using a magnetically purified combination of CD34+ and CD133+ SCs. This new method was shown to be safe on the patients, who also showed moderate neurological improvements in the long run within the 5-year follow-up period. We were able to simultaneously purify three different populations of viable and functional SCs (CD34+, CD133+, and CD34+/CD133+ SCs) that might have varying curative potentials, for transplantation into patients with complete SCIs.

We showed that the cells we used in this therapy were both highly pure and viable. ${ }^{14}$ Also, preliminary trials in ALS patients have determined that the intervention of transforming SCs into new neural support cells is safe, and researchers have begun testing whether it can slow the decline or improve symptoms. ${ }^{19}$ However, before SC therapy can be applied in a clinical setting for any of the neurodegenerative disorders, intensive research must be completed to fully understand $\mathrm{SC}$ behavioral changes and interaction when placed in a neurological physiological microenvironment that mimics the original body environment.

\section{Conclusion}

In conclusion, our results indicate that human adult BM HSCs may have the potential to differentiate into cells with neural-like phenotypes. Subsequently, isolated CD34+ SC populations represent a source of readily available SCs capable of differentiating into astrocytes, CD133+ into neurons, and CD34+/CD133+ into oligodendrocytes, which may aid in generating neurons for transplantation therapy in CNS injuries and neurodegenerative diseases. Nevertheless, translation to the bedside remains a long way off.

\section{Acknowledgments}

This work was supported by Stem Cells of Arabia, Jordan, Orthopedic and Spinal Center, Jordan, and the National Center for Diabetes, Endocrinology and Genetics, Amman, Jordan.

\section{Disclosure}

The authors report no conflicts of interest in this work.

\section{References}

1. Chipman PH, Toma JS, Rafuse VF. Generation of motor neurons from pluripotent stem cells. Prog Brain Res. 2012;201:313-331.

2. Alsanie WF, Niclis JC, Petratos S. Human embryonic stem cellderived oligodendrocytes: protocols and perspectives. Stem Cells Dev. 2013;22(18):2459-2476.

3. Kim KS, Kim HS, Park JM, et al. Long-term immunomodulatory effect of amniotic stem cells in an Alzheimer's disease model. Neurobiol Aging. 2013;34(10):2408-2420.

4. Li JF, Zhang DJ, Geng T, et al. The potential of human umbilical cord-derived mesenchymal stem cells as a novel cellular therapy for multiple sclerosis. Cell Transplant. 2014;23(Suppl 1): S113-S122.

5. Zhao P, Luo Z, Tian W, et al. Solving the puzzle of Parkinson's disease using induced pluripotent stem cells. Exp Biol Med (Maywood). 2014; 239(11):1421-1432.

6. Rossignol J, Fink KD, Crane AT, et al. Reductions in behavioral deficits and neuropathology in the R6/2 mouse model of Huntington's disease following transplantation of bone-marrow-derived mesenchymal stem cells is dependent on passage number. Stem Cell Res Ther. 2015;6:9.

7. Ismail R, Allaudin ZN, Lila MA. Scaling-up recombinant plasmid DNA for clinical trial: current concern, solution and status. Vaccine. 2012;30(41):5914-5920.

8. Zhang WY, de Almeida PE, Wu JC. Teratoma formation: a tool for monitoring pluripotency in stem cell research. In: The Stem Cell Research Community, editor. StemBook. Cambridge (MA): Harvard Stem Cell Institute; 2008.

9. Bidwell JP, Alvarez MB, Hood M, Jr, Childress P. Functional impairment of bone formation in the pathogenesis of osteoporosis: the bone marrow regenerative competence. Curr Osteoporos Rep. 2013;11(2):117-125.

10. Radtke CL, Nino-Fong R, Esparza Gonzalez BP, Stryhn H, McDuffee LA. Characterization and osteogenic potential of equine muscle tissue- and periosteal tissue-derived mesenchymal stem cells in comparison with bone marrow- and adipose tissue-derived mesenchymal stem cells. Am J Vet Res. 2013;74(5):790-800

11. Wegmeyer H, Broske AM, Leddin M, et al. Mesenchymal stromal cell characteristics vary depending on their origin. Stem Cells Dev. 2013;22(19):2606-2618. 
12. Fuss IJ, Kanof ME, Smith PD, Zola H. Isolation of whole mononuclear cells from peripheral blood and cord blood. Curr Protoc Immunol. 2009; Chapter 7:Unit7.1.

13. Jamous M, Al-Zoubi A, Khabaz MN, Khaledi R, Al Khateeb M, Al-Zoubi Z. Purification of mouse bone marrow-derived stem cells promotes ex vivo neuronal differentiation. Cell Transplant. 2010; 19(2):193-202.

14. Al-Zoubi A, Jafar E, Jamous M, et al. Transplantation of purified autologous leukapheresis-derived CD34+ and CD133+ stem cells for patients with chronic spinal cord injuries: long-term evaluation of safety and efficacy. Cell Transplant. 2014;23(Suppl 1):S25-S34.

15. Deng J, Petersen BE, Steindler DA, Jorgensen ML, Laywell ED. Mesenchymal stem cells spontaneously express neural proteins in culture and are neurogenic after transplantation. Stem Cells. 2006;24(4):1054-1064.
16. Hermann A, Gastl R, Liebau S, et al. Efficient generation of neural stem cell-like cells from adult human bone marrow stromal cells. J Cell Sci. 2004;117(Pt 19):4411-4422.

17. Abo-Grisha N, Essawy S, Abo-Elmatty DM, Abdel-Hady Z. Effects of intravenous human umbilical cord blood CD34+ stem cell therapy versus levodopa in experimentally induced Parkinsonism in mice. Arch Med Sci. 2013;9(6):1138-1151.

18. Sigurjonsson OE, Perreault MC, Egeland T, Glover JC. Adult human hematopoietic stem cells produce neurons efficiently in the regenerating chicken embryo spinal cord. Proc Natl Acad Sci US A. 2005;102(14):5227-5232.

19. Thomsen GM, Gowing G, Svendsen S, Svendsen CN. The past, present and future of stem cell clinical trials for ALS. Exp Neurol. 2014; 262(Pt B): 127-137.

\section{Publish your work in this journal}

The Journal of Neurorestoratology is an international, peer-reviewed, open access online journal publishing original research and review articles on the subject of Neurorestoratology. To provide complete coverage of this revolutionary field the Journal of Neurorestoratology will report on relevant experimental research, technological advances, and

\section{Dovepress}

clinical achievements. The manuscript management system is completely online and includes a very quick and fair peer-review system, which is all easy to use. Visit http://www.dovepress.com/testimonials.php to read real quotes from published authors.

Submit your manuscript here: http://www.dovepress.com/journal-of-neurorestoratology-journal 